\title{
ON THE NATURE OF OPTICAL IMAGES.
}

By Alber't B. Porter.

$\mathrm{T}$

HERE is no doubt that the teaching of mathematics has suffered from the somewhat arbitrary division of the subject matter into what have been aptly termed "water-tight compartments." To a lesser degree the same thing is true of physics, if current teaching may be judged by some of the current texts. Thus in the treatment of light, for example, although the sharp distinction between geometrical and physical optics has largely passed with the passing of the old-fashioned (but often indispensable) ray-method of treating the formation of images, yet the wavemethod which has replaced it is sometimes presented in a form almost as purely geometrical. We have abandoned the diagrams in which the so-called rays were frankly intended to represent nothing more than directions of propagation, and have substituted figures showing an impossible array of sharply limited wave-fronts primly marching toward a focus, with never a trace of diffractive fraying at the edges. Even in the few text-books with which I am acquainted in which this fraying is taken into account in an investigation of the effects of aperture on resolving power, the discussion follows so far after the study of image formation that the student must almost inevitably absorb the idea that diffraction and interference merely act to modify an image otherwise formed. Nor do I anywhere recall in the elementary books an explicit statement to the effect that all optical images arise by interference and are indeed particular cases of interference patterns. That this is, however, true appears at once from a consideration of the method of propagation of light by means of secondary wavelets, which spread in all directions from every point of the wave-front in such a manner that, were it not for interference, each point in the image would receive light from all points in the object. Dark places in the image are thus dark because of destructive interference, and light parts are bright on 
account of additive interference. All this, though of course sufficiently familiar to the teacher, may be far from evident to the student, and this is my excuse for describing a simple and quickly arranged experiment which shows the relation between interference and image formation in a manner so striking as not to be easily forgotten.

The experiment consists in passing a parallel beam of monochromatic light through a coarsely ruled, black-line, diffraction grating, and then through a convex lens. On the far side of the lens a system of sharply defined interference fringes is formed which can be seen by aid of an eyepiece, or intercepted on a screen, at any point over a considerable range along the axis. Somewhere in this system of fringes is the geometrical image of the grating, but it is visually quite indistinguishable from any other transverse section of the fringe system. Clearly in this case, the geometrical image is merely that section in which the geometrical condition of similarity to the object is satisfied.

The best arrangement of the experiment is the following. Light from an arc lamp $A$ is focused by means of the lens $B$ upon a narrow slit $C$. Thence it passes through a direct-vision prism at $D$,

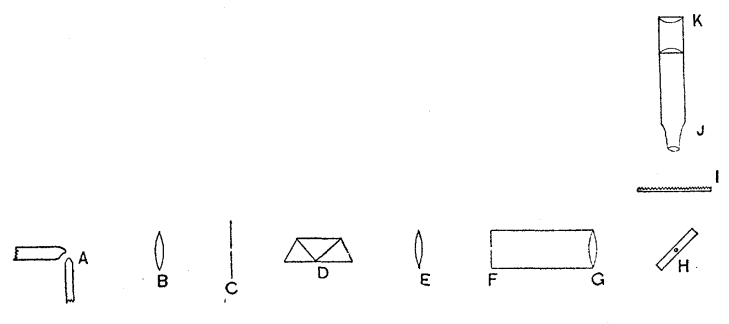

Fig. 1.

and the spectrum is focused by the lens $E$ upon the narrow slit of a collimator $F G$. The parallel beam of monochromatic light thus obtained falls upon the mirror $H$ of a microscope $K J$, upon whose stage, at $I$, the grating is placed in such a position that its ruled lines are parallel to the projections of the two slits $C$ and $F$. Using a black-line grating of 400 lines to the inch, and having both slits narrowed down to a small fraction of a millimeter so as to secure very homogeneous illumination, the field of view was examined 
with $2 / 3$ inch objective and $I$ inch eyepiece. The interference fringes appeared in the field of the eyepiece with exquisitely sharp definition throughout the whole range of the coarse adjustment of the microscope, $i$. $e$., over a distance of $58 \mathrm{~mm}$., beginning with the front of the objective in contact with the grating and with its focal plane $7 \mathrm{~mm}$. below the ruled surface; and the fringes could be traced through a much greater range by withdrawing the eyepiece and moving it back along the axis. As the microscope is slowly focused upward, the bands undergo curious changes in appearance, the lines showing sometimes close together and again farther apart, but the definition is almost equally sharp throughout the whole range of adjustment, so that any section of the fringe system is as good an apparent image as any other section. Similar but less perfect effects may be obtained by illuminating the field by means of sodium light passing through a slit a couple of millimeters wide at a distance of one or two meters.

If the angle of the incidence of the light on the grating is changed by moving the mirror, the whole fringe system shifts to one side or the other except in the focal plane, where it remains stationary. This shows (I) that the focal plane is the plane in which the interference fringes formed by light of all incidences coincide ; $(2)$ that, when a broad source is used, the geometrical image is really a superposition of coincident interference patterns; and (3) that the usual absence of a sharp image outside the focal plane is due to the more or less uniform illumination resulting from the overlapping of fringe systems due to light coming from various points in the source. When the grating is illuminated by a parallel beam of white light by means of a collimator with very narrow slit or, less perfectly, by a distant gas flame turned edgewise, the effects are similar except that outside the focal plane the fringes are colored. Hence (4) the focal plane is also the plane of achromatic interference, i. e., the plane in which the fringes due to light of various wave-lengths coincide.

These experiments show very clearly why it is in general essential to use a condenser to illuminate the field of a microscope in order to obtain a critical image, i.e., an image which comes sharply into and out of focus and which is hence as free as possible from confusion with details of structure lying above and below the focal 
plane. It is interesting to observe how, as the illumination is made less homogeneous and more convergent, the distance through which the interference bands can be distinguished decreases, and the precision of focusing increases.

In the case of self-luminous objects, although the geometrical image is still to be considered as an interference pattern, the effects outside of the focal plane are greatly modified by the absence of definite phase relations between the waves emanating from various points. 